PROCEEDINGS OF THE

AMERICAN MATHEMATICAL SOCIETY

Volume 128, Number 12, Pages 3629-3637

S 0002-9939(00)05506-4

Article electronically published on June 7, 2000

\title{
POINTWISE CONVERGENCE OF A CLASS OF NON-ORTHOGONAL WAVELET EXPANSIONS
}

AHMED I. ZAYED

(Communicated by Christopher D. Sogge)

\begin{abstract}
Non-orthogonal wavelet expansions associated with a class of mother wavelets is considered. This class of wavelets comprises mother wavelets that are not necessarily integrable over the whole real line, such as Shannon's wavelet. The pointwise convergence of these wavelet expansions is investigated. It is shown that, unlike other wavelet expansions, the ones under consideration do not necessarily converge pointwise to the functions at points of continuity, unless a more stringent condition, such as bounded variation, is imposed.
\end{abstract}

\section{INTRODUCTION}

Wavelet expansions have been the focus of many research papers in the last few years. One of the reasons for their popularity is that they provide a more efficient representation of functions than other orthogonal expansions. The orthogonal wavelet expansion of a function in $L^{2}(\mathcal{R})$ is known to converge to it in the sense of $L^{2}(\mathcal{R})$, but the pointwise convergence is a little bit more subtle. Y. Meyer [4, Ch. 2 ] was among the first to study the convergence of orthogonal wavelet expansions. He showed that if the mother wavelet is $r$-regular, the orthogonal wavelet expansion of a function will converge to it in the sense of $L^{p}(\mathcal{R}), 1 \leq p<\infty$, and in the sense of some Sobolov spaces as well.

A function $f(x), x \in \mathcal{R}^{d}, d \geq 1$, is said to be $r$-regular (in the sense of Meyer) if

$$
\left|D^{\alpha} f(x)\right| \leq \frac{C_{\alpha, m}}{(1+|x|)^{m}},
$$

for all $\alpha$ with $|\alpha| \leq r$ and $m=0,1,2, \cdots$, where $C_{\alpha, m}$ are constants. Here $\alpha=$ $\left(\alpha_{1}, \cdots, \alpha_{d}\right)$ is a multi-index with $\alpha_{i}(i=1, \cdots, d)$ being a non-negative integer and $|\alpha|=\sum_{i=1}^{d} \alpha_{i}$, and

$$
D^{\alpha}=\frac{\partial^{|a|}}{\partial x_{1}^{\alpha_{1}} \cdots \partial x_{d}^{\alpha_{d}}} .
$$

Before we state some important results on pointwise convergence of wavelet expansions, let us recall the following definition.

Received by the editors June 16, 1998 and, in revised form, February 24, 1999.

1991 Mathematics Subject Classification. Primary 42C15, 42C05; Secondary 40A05, 40A30.

Key words and phrases. Non-orthogonal wavelets, Shannon's wavelet, wavelet expansions, pointwise convergence. 
Definition 1. A multiresolution analysis of $\mathcal{R}^{d}$ is a decomposition of $L^{2}\left(\mathcal{R}^{d}\right)$ into a nested sequence of closed subspaces $\left\{V_{i}\right\}_{n=-\infty}^{\infty}$ such that

1) $\ldots \subset V_{-1} \subset V_{-2} \subset V_{0} \subset V_{1} \subset V_{2} \subset \cdots$,

2) $\bigcup_{i=-\infty}^{\infty} V_{i}$ is dense in $L^{2}\left(\mathcal{R}^{d}\right)$,

3) $\bigcap_{i=-\infty}^{\infty} V_{i}=\{0\}$,

4) $f(x) \in V_{i}$ if and only if $f(2 x) \in V_{i+1}$, for all $i$,

5) $V_{0}$ is closed under integer translation, i.e., $f(x) \in V_{0}$ implies that $f(x-k) \in$ $V_{0}$, for all $k=\left(k_{1}, \cdots, k_{d}\right)$ with $k_{i}$ being a nonnegative integer,

6) there exists a function $\phi(x) \in V_{0}$, called the scaling function or the father wavelet, such that $\left\{\phi_{k}(x)=\phi(x-k)\right\}$ is an orthonormal basis for $V_{0}$.

A multiresolution analysis is said to be $r$-regular if its scaling function is $r$-regular.

Let $W_{j}$ denote the orthogonal complement of $V_{j}$ in $V_{j+1}$. It is known that there is a family $\left\{\psi^{\lambda}(x)\right\}_{\lambda \in \Lambda}$ (whose cardinality depends on the dimension $d$ ) of basic mother wavelets such that $\left\{\psi_{m, n}^{\lambda}(x)=2^{m d / 2} \psi^{\lambda}\left(2^{m} x-n\right)\right\}$ is an orthonormal basis of $W_{m}$, for fixed $m$, where $m$ is an integer, $n$ is a multi-integer, and $\lambda \in \Lambda$, and is an orthonormal basis of $L^{2}\left(\mathcal{R}^{d}\right)$, for all $m$ and $n$. In one dimension the cardinality of $\Lambda$ is one; hence there is one basic mother wavelet $\psi$ generating an orthonormal basis of $L^{2}(\mathcal{R})$.

From the definition, it follows by induction that

$$
L^{2}\left(\mathcal{R}^{d}\right)=\bigoplus_{i=-\infty}^{\infty} W_{i}
$$

where $\oplus$ denotes the direct sum and

$$
L^{2}\left(\mathcal{R}^{d}\right)=V_{0} \bigoplus_{i=0}^{\infty} W_{i}
$$

It also follows that in one dimension $\left\{\phi_{m, n}(x)=2^{m / 2} \phi\left(2^{m} x-n\right)\right\}_{n=-\infty}^{\infty}$ is an orthonormal basis for $V_{m}$, for each $m$. From (11) and (2), it follows that

$$
f(x)=\sum_{j ; k ; \lambda} a_{j, k}^{\lambda} \psi_{j, k}^{\lambda}(x),
$$

where $a_{j, k}^{\lambda}$ are the $L^{2}$ expansion coefficients of $f$ and

$$
f(x)=\sum_{k} b_{k} \phi_{k}(x)+\sum_{j \geq 0 ; k ; \lambda} a_{j, k}^{\lambda} \psi_{j, k}^{\lambda}(x),
$$

where $b_{k}$ and $a_{j, k}^{\lambda}$ are the $L^{2}$ expansion coefficients of $f$. The convergence of the above series is in the sense of $L^{2}$. In one dimension, we have

$$
f(x)=\sum_{k} b_{m, k} \phi_{m, k}+\sum_{j \geq m ; k} a_{j, k} \psi_{j, k}(x)=f_{m}(x)+r_{m}(x) .
$$

The function $f_{m}$ is in the space $V_{m}$ and in fact is the projection of $f$ onto $V_{m}$.

Assuming that the scaling function $\phi$ of the multiresolution analysis is $r$-regular, G. Walter [6] proved that the orthogonal wavelet expansion of a function $f \in L^{2} \cap L^{1}$ converges to $f$ pointwise at every point of continuity of $f$ and uniformly on compact 
subsets of any interval $(a, b)$ on which $f$ is continuous. Later, he relaxed this condition and assumed that $\phi$ satisfies the condition

$$
|\phi(x)| \leq \frac{C}{(1+|x|)^{3}} .
$$

In 2, 3], S. Kelly, M. Kon, and L. Raphael improved Walter's results by proving pointwise convergence of orthogonal wavelet expansions not only under less stringent conditions, but also by extending them to $n$ dimensions. Crucial to their proofs is the following definition.

Definition 2. A function $f(x)$ on $\mathcal{R}^{d}$ is radial if it depends on $|x|$ only. A realvalued radial function is radial decreasing if $f(x) \leq f(y)$ whenever $|y| \leq|x|$. A function $f(x)$ is in the class $\mathcal{R B}$ if it is absolutely bounded by an $L^{1}$ radial decreasing function $\eta(x)$, i.e., with $\eta\left(x_{1}\right)=\eta\left(x_{2}\right)$ whenever $\left|x_{1}\right|=\left|x_{2}\right|$ and with $\eta\left(x_{1}\right) \leq \eta\left(x_{2}\right)$ whenever $\left|x_{1}\right| \geq\left|x_{2}\right|$, and $\eta(x) \in L^{1}\left(\mathcal{R}^{d}\right)$.

They showed, among other things, that if $\phi, \psi^{\lambda}$ are in the class $\mathcal{R B}$ for all $\lambda$, then the series (3) and (4) of any function $f \in L^{p}\left(\mathcal{R}^{d}\right)$ converge to $f$ pointwise almost everywhere. Moreover, if only $\psi^{\lambda} \ln (2+|x|) \in \mathcal{R B}$, then the wavelet expansion (3) of any function $f \in L^{p}\left(\mathcal{R}^{d}\right)$ converges to $f$ pointwise almost everywhere.

In all the work cited above on pointwise convergence it is essential that the summation kernel of the wavelet series

$$
P_{m}(x, y)=\sum_{j<m ; k ; \lambda} \psi_{j, k}^{\lambda}(x) \bar{\psi}_{j, k}^{\lambda}(y)=\sum_{k} \phi_{m, k}(x) \bar{\phi}_{m, k}(y)
$$

be absolutely bounded by a dilation of an $L^{1}$ - radially decreasing convolution kernel, i.e., by $H\left(2^{m}|x-y|\right)$, where $H \in \mathcal{R B}$. There are, however, some mother wavelets that do not satisfy these conditions. Chief among them is Shannon's wavelet, which is given by

$$
\psi(x-1 / 2)=\frac{\sin 2 \pi t-\sin \pi x}{\pi x},
$$

and for which the associated scaling function $\phi$ is given by $\phi(x)=\sin \pi x / \pi x$. The summation kernels in the subspaces $V_{0}$ and $W_{0}$ are easily seen to be

$$
\frac{\sin \pi(x-y)}{\pi(x-y)}=\sum_{k=-\infty}^{\infty} \frac{\sin \pi(x-k)}{\pi(x-k)} \frac{\sin \pi(y-k)}{\pi(y-k)},
$$

and

$$
\begin{aligned}
& \frac{\sin 2 \pi(x-y)-\sin \pi(x-y)}{\pi(x-k)}= \\
& \quad \sum_{k=-\infty}^{\infty} \frac{\sin 2 \pi(x-k)-\sin \pi(x-k)}{\pi(x-k)} \frac{\sin 2 \pi(y-k)-\sin \pi(y-k)}{\pi(y-k)} .
\end{aligned}
$$

Equation (6) is well known, but (7), to the best of our knowledge, is less known. From (6) and (7) it is clear that neither kernel is in $L^{1}(\mathcal{R})$; hence neither one can be absolutely bounded by an $L^{1}(\mathcal{R})$ radially decreasing function.

Of course, it is easy to study directly the pointwise convergence of the Shannon wavelet series, but this is a very special case and of no great interest. Instead, we shall study the pointwise convergence of wavelet expansions associated with a class of mother wavelets that contains Shannon's wavelet as a special case. We shall assume that neither the scaling function of the multiresolution analysis, $\phi$, 
nor the mother wavelet $\psi$ is in $L^{1}$, and we shall not assume that they generate an orthonormal basis of $V_{0}$ or of $W_{0}$. This means that unlike the wavelet expansions studied in [2, 3, 6], ours are not necessarily orthonormal. Therefore, we replace condition (6) of Definition [1] by the weaker condition that $\{\phi(x-k)\}$ is a Riesz basis of $V_{0}$.

The foregoing discussion may give a false impression that our results are stronger than those of [2, 3, 6], while in fact they are neither stronger nor weaker, but complementary. On the one hand, we do not assume that $\phi$ or $\psi$ is integrable or generates an orthonormal basis, but on the other hand we assume a stronger condition on $\phi$, namely, that its Fourier transform $\hat{\phi}$ has compact support. By not requiring that $\phi$ or $\psi$ be absolutely integrable, the partial sums of the wavelet series behave differently from those studied in [2, 3, 6]. The continuity of a function $f$ is no longer sufficient to ensure that its wavelet series expansion will converge to it pointwise. A more stringent condition, such as bounded variation, is needed.

\section{The MAIn Result}

We adopt the following standard notation. The Fourier transform of a function $f(x)$ is defined as

$$
\hat{f}(\omega)=\frac{1}{\sqrt{2 \pi}} \int_{-\infty}^{\infty} f(x) e^{i \omega x} d x,
$$

so that the inverse transform is given by

$$
f(x)=\frac{1}{\sqrt{2 \pi}} \int_{-\infty}^{\infty} \hat{f}(\omega) e^{-i x \omega} d \omega,
$$

whenever the integrals exist. A summation of the form $\sum_{k}$ is understood to mean $\sum_{k=-\infty}^{\infty}$. The characteristic function of a set $A$ will be denoted by $\chi_{A}(x)$, i.e., $\chi_{A}(x)=1$ if $x \in A$ and zero otherwise. The space of all locally integrable functions on $\mathcal{R}$ will be denoted by $L_{\text {loc }}^{1}(\mathcal{R})$.

Definition 3. Let $\mathcal{A}$ be the set of all scaling functions $\phi \in L^{2}(\mathcal{R})$ that satisfy the following conditions:

1) $\hat{\phi}(\omega)$ has support in some interval $[-a, a], 0<a \leq \pi$, and $|\phi(x)| \leq c /(|x|+1)$;

2) there exist two positive numbers $A$ and $B$ such that

$$
0<A \leq \sum_{k}|\hat{\phi}(\omega+2 \pi k)|^{2} \leq B<\infty ;
$$

3) $\phi(x)$ satisfies the dilation equation

$$
\phi(x)=\sum_{k} a_{k} \phi(2 x-k),
$$

for some sequence $\left\{a_{k}\right\}$ with $\sum_{k}\left|a_{k}\right|^{2}<\infty$;

4) $\hat{\phi}(\omega)$ is bounded and continuous near zero with $\hat{\phi}(0) \neq 0$;

$5)$ the support of $\hat{\phi}$, which will be denoted by $E$, is of the form

$$
E=\bigcup_{i=1}^{N}\left[a_{i}, b_{i}\right] \cup O
$$

where $O$ is a set of measure zero.

The class of mother wavelets associated with the class $\mathcal{A}$ will be called the class of Shannon-type wavelets and will be denoted by $\mathcal{A}_{W}$. 
In addition to Shannon's wavelet, the class $\mathcal{A}_{W}$ contains infinitely many functions; among them, for example, is the function

$$
\psi(x-1 / 2)=\frac{\sin 2 \pi t+\sin \pi t-2 \sin (3 \pi t / 2)}{\pi t},
$$

which is associated with the scaling function $\phi(t)=[2 \sin (\pi t / 2)-\sin \pi t] / \pi t$, or

$$
\hat{\phi}(\omega)= \begin{cases}1, & |\omega|<\pi / 2 \\ -1, & \pi / 2<|\omega|<\pi\end{cases}
$$

(for more examples of wavelets in the class $\mathcal{A}_{W}$, see [7]).

Let us define $V_{0}$ as the closed linear span of the set $\{\phi(x-k)\}$ and likewise $V_{m}$ as the closed linear span of $\left\{\phi_{m, k}=2^{m / 2} \phi\left(2^{m} x-k\right)\right\}$. The space $V_{0}$ is the same as the space $B_{E}$ of functions bandlimited to $E$ since any $f \in B_{E}$ can be written in the form $\hat{f}=\hat{g} \hat{\phi}$, where $\hat{g}$ is the $2 \pi$-periodic extension of $\hat{f} / \hat{\phi}$. Condition (2) implies that $\{\phi(x-k)\}$ is a Riesz basis of $V_{0}$. Condition (3) implies that the spaces $V_{m}$ have the nested property (1) of Definition 1 . Conditions (2) and (3) ensure that the set $\phi_{m, k}$ is a Riesz basis of $V_{m}$ for each $m$ and that $\bigcap_{m} V_{m}=\{0\}$. Condition (4) ensures that property (2) of Definition 1 1 holds; see [1, p. 141-142].

Therefore, Conditions (1)-(4) guarantee that we have a multiresolution analysis and a mother wavelet $\psi(x)$. Without loss of generality, we assume that $\hat{\phi}(0)=1$. Condition (5) implies that if

$$
\begin{aligned}
q(x)=\frac{1}{\sqrt{2 \pi}} \int_{E} e^{-i x \omega} d \omega & =\frac{1}{\sqrt{2 \pi}} \sum_{k=1}^{N} \int_{a_{k}}^{b_{k}} e^{-i x \omega} d \omega \\
& =\frac{1}{\sqrt{2 \pi}} \sum_{k=1}^{N} \frac{e^{-i a_{k} x}-e^{-i b_{k} x}}{i x}
\end{aligned}
$$

then

$$
\lambda q(\lambda x)=\frac{1}{\sqrt{2 \pi}} \sum_{k=1}^{N} \frac{e^{-i a_{k} \lambda x}-e^{-i b_{k} \lambda x}}{i x},
$$

which is clearly bounded for all $x$ and $\lambda$. Since $\{\phi(x-k)\}$ is a Riesz basis of $V_{0}$, it has a biorthonormal basis $\left\{\phi_{k}^{*}(x)\right\}$ such that for any $f \in V_{0}$ we have

$$
f(x)=\sum_{k}\left\langle f, \phi_{k}^{*}\right\rangle \phi_{0, k}(x),
$$

in the sense of $L^{2}(\mathcal{R})$, where $\phi_{0, k}(x)=\phi(x-k)$. Similarly, for any $f \in V_{m}$ and fixed $m$, we have

$$
f(x)=\sum_{k}\left\langle f, \phi_{m, k}^{*}\right\rangle \phi_{m, k}(x),
$$

where $\left\{\phi_{m, k}^{*}(x)\right\}_{k=-\infty}^{\infty}$ is the biorthonormal basis of $\left\{\phi_{m, k}(x)\right\}_{k=-\infty}^{\infty}$. From the relation

$$
\left\langle\phi_{n}, \phi_{k}^{*}\right\rangle=\delta_{k, n}=\int_{-\infty}^{\infty} \phi(x-n) \overline{\phi^{*}}{ }_{k}(x) d x
$$

we obtain by a change of variable that

$$
\left\langle\phi_{n}, \phi_{k}^{*}\right\rangle=\delta_{k, n}=2^{m} \int_{-\infty}^{\infty} \phi\left(2^{m} x-n\right){\overline{\phi^{*}}}_{k}\left(2^{m} x\right) d x .
$$


It follows that $\left\{2^{m / 2} \phi_{k}^{*}\left(2^{m} x\right)\right\}_{k=-\infty}^{\infty}$ is the biorthonormal basis of $\left\{\phi_{m, k}(x)\right\}_{k=-\infty}^{\infty}$ for each $m$.

We recall the following version of the second mean-value theorem: If $f$ is increasing and $h$ is continuous on $J=[a, b]$, then there exists $c \in J$ such that

$$
\int_{a}^{b} f(x) h(x) d x=f(a) \int_{a}^{c} h(x) d x+f(b) \int_{c}^{b} h(x) d x .
$$

Now we can state and prove our main theorem.

Theorem 1. Let $\phi \in \mathcal{A}, \phi_{k}^{*}(x)=O\left(k^{-\alpha}\right)$ as $k \rightarrow \infty$ for some $\alpha>0$, and $f \in$ $L^{2}(\mathcal{R})$. Assume that either

1) $f \in L^{1}(\mathcal{R})$ and of bounded variation on every bounded interval, or

2) $f \in L_{\text {loc }}^{1}(\mathcal{R})$, of bounded variation in neighborhoods of $\pm \infty$ and tends to zero as $|x| \rightarrow \infty$,

then the wavelet series

$$
f_{m}(x)=\sum_{k}\left\langle f, \phi_{m, k}^{*}\right\rangle \phi_{m, k}(x)
$$

converges to $f(x)$ as $m \rightarrow \infty$ at every point of continuity of $f(x)$.

The result is best possible in the sense that the continuity of a function at a point is not sufficient to ensure the convergence of its wavelet expansion.

Proof. From Eq. (5), it is clear that the partial sum $\sum_{j<m, k} a_{j, k} \psi_{j, k}(x)$ of the wavelet expansion of $f$ is the same as the projection $f_{m}(x)$ of $f$ on $V_{m}$. Therefore, it suffices to study the convergence of $f_{m}(x)$ as $m \rightarrow \infty$.

The space $V_{0}$ is a reproducing-kernel Hilbert space since point evaluation is continuous:

$$
|f(x)|=\left|\frac{1}{\sqrt{2 \pi}} \int_{E} \hat{f}(\omega) e^{-i x \omega} d \omega\right| \leq C\|\hat{f}\|=C\|f\| .
$$

Therefore, it has a reproducing kernel $k(t, x)$, which is easily seen to be a convolution kernel. For, if $f \in V_{0}$, then

$$
f(x)=\frac{1}{\sqrt{2 \pi}} \int_{E} \hat{f}(\omega) e^{-i x \omega} d \omega=\int_{-\infty}^{\infty} f(t) k(t, x) d t
$$

where

$$
k(t, x)=\frac{1}{2 \pi} \int_{E} e^{i \omega(t-x)} d \omega=k(t-x) .
$$

The last integral in (9) is absolutely convergent by the Cauchy-Schwarz inequality because both $f$ and $k$ are in $L^{2}(\mathcal{R})$. The reproducing kernel has also the representation $k(t, x)=\sum_{n} \phi_{n}^{*}(t) \phi(x-n)$, where the series converges in the sense of $L^{2}$. But from the hypothesis, the series also converges absolutely and uniformly for all $x$ and $t$ to a function $q_{0}(t, x)$. Thus, by standard arguments, $q_{0}(t, x)=k(t-x)$ almost everywhere. One can also show directly that if $f \in V_{0}$, then

$$
\begin{aligned}
\int_{-\infty}^{\infty} f(t) q_{0}(t, x) d t & =\sum_{n}\left(\int_{-\infty}^{\infty} f(t) \phi_{n}^{*}(t) d t\right) \phi(x-n) \\
& =\sum_{n}\left\langle f, \phi_{n}^{*}\right\rangle \phi(x-n)=f(x),
\end{aligned}
$$

since strong convergence implies weak convergence and, in addition, strong convergence in a reproducing-kernel Hilbert space implies pointwise convergence, as well as uniform convergence on any set on which $k(x, x)$ is uniformly bounded. 
The projection of $f \in L^{2}(\mathcal{R})$ on $V_{0}$ is

$$
f_{0}(x)=\int_{-\infty}^{\infty} f(t) q_{0}(t, x) d t
$$

and similarly by Property 1) of Definition 1, we have that the projection of $f \in$ $L^{2}(\mathcal{R})$ on the space $V_{m}$ is given by

$$
f_{m}(x)=\int_{-\infty}^{\infty} f(t) q_{m}(t, x) d t
$$

where

$$
q_{m}(t, x)=\sum_{n} \phi_{m, n}^{*}(t) \phi_{m, n}(x)=2^{m} \sum_{n} \phi_{n}^{*}\left(2^{m} t\right) \phi\left(2^{m} x-n\right) .
$$

Thus, $q_{m}(t, x)=2^{m} q_{0}\left(2^{m} t, 2^{m} x\right)$. Setting $\lambda=2^{m}$, we obtain

$$
f_{m}(x)=f_{\lambda}(x)=\lambda \int_{-\infty}^{\infty} f(t) q_{0}(\lambda t, \lambda x) d t .
$$

Furthermore, we have (5. p. 25])

$$
\chi_{E}(\omega)=\int_{-\infty}^{\infty} q_{0}(z) e^{-i \omega z} d z
$$

which implies, in view of Condition 4 of Definition 3, that

$$
\lambda \int_{-\infty}^{\infty} q_{0}(\lambda(t-x)) d t=\int_{-\infty}^{\infty} q_{0}(z) d z=\chi_{E}(0)=1
$$

From Equations (10) and (11), we obtain

$$
\begin{aligned}
f_{\lambda}(x)-f(x) & =\lambda \int_{-\infty}^{\infty}[f(t)-f(x)] q_{0}(\lambda(t-x)) d t \\
& =\lambda \int_{-\infty}^{\infty}[f(x+\eta)-f(x)] q_{0}(\lambda \eta) d \eta=I_{1}(x, \lambda)+I_{2}(x, \lambda),
\end{aligned}
$$

where

$$
I_{1}(x, \lambda)=\int_{-\infty}^{0}, \quad \text { and } \quad I_{2}(x, \lambda)=\int_{0}^{\infty} .
$$

We shall only show that $\lim _{\lambda \rightarrow \infty} I_{2}(x, \lambda)=0$ since the proof for $I_{1}$ is similar.

Let $I_{2}=I_{3}+I_{4}+I_{5}-I_{6}$, where

$$
\begin{gathered}
I_{3}=\lambda \int_{0}^{\delta}[f(x+\eta)-f(x)] q_{0}(\lambda \eta) d \eta, \\
I_{4}=\lambda \int_{\delta}^{A}[f(x+\eta)-f(x)] q_{0}(\lambda \eta) d \eta, \\
I_{5}=\lambda \int_{A}^{\infty} f(x+\eta) q_{0}(\lambda \eta) d \eta, \quad \text { and } \quad I_{6}=\lambda \int_{A}^{\infty} f(x) q_{0}(\lambda \eta) d \eta,
\end{gathered}
$$

where $\delta$ and $A$ are to be determined later on. Let $\epsilon>0$ be given. Since

$$
I_{6}=\lambda f(x) \int_{A}^{\infty} q_{0}(\lambda \eta) d \eta=f(x) \int_{\lambda A}^{\infty} q_{0}(y) d y,
$$

and

$$
\int_{0}^{\infty} q_{0}(y) d y<\infty
$$


it follows that by choosing $\lambda$ large enough, we can make $\left|I_{6}\right| \leq \epsilon / 6$. From the relation

it follows that

$$
q_{0}(y)=\frac{1}{2 \pi} \int_{E} e^{i \omega y} d \omega
$$

$$
\lambda \int_{a}^{b} q_{0}(\lambda y) d y=\frac{1}{2 i \pi} \int_{-\infty}^{\infty} e^{i a \omega \lambda}\left\{\chi_{E}(\omega) \frac{e^{i \omega \lambda(b-a)}-1}{\omega}\right\} d \omega
$$

for any finite numbers $a, b$. But since the function in the curly brackets is integrable, it follows that $\left|\lambda \int_{a}^{b} q_{0}(\lambda y) d y\right|$, or equivalently, $\left|\int_{a \lambda}^{b \lambda} q_{0}(z) d z\right|$ is bounded by, say $L$, for all $\lambda$. Moreover, by (12), we have

$$
\lambda \int_{a}^{b} q_{0}(\lambda y) d y=\int_{a \lambda}^{b \lambda} q_{0}(z) d z \rightarrow 0
$$

as $\lambda \rightarrow \infty$ for any $b>a>0$.

If $f \in L^{1}(\mathcal{R})$, then in view of (8) we can choose $A$ large enough so that

$$
\left|I_{5}\right| \leq C \int_{A}^{\infty}|f(x+\eta)| d \eta \leq \epsilon / 6
$$

Because $f$ is assumed to be of bounded variation, we can assume, without loss of generality, that it is monotone increasing, and set $g(\eta)=f(x+\eta)-f(x)$. Clearly, $g$ is monotone increasing with $g(0)=0$. And since $q_{0}$ is continuous, being the Fourier transform of an integrable function $\chi_{E}$, it follows from the second meanvalue theorem

$$
I_{3}=g(\delta) \int_{c \lambda}^{\delta \lambda} q_{0}(y) d y
$$

for some $c$ with $0<c<\delta$. By choosing $\delta$ small enough so that $|g(\delta)| \leq \epsilon /(3 L)$, we obtain that $\left|I_{3}\right| \leq \epsilon / 3$.

Having determined $\delta$ and $A$ we can now estimate $I_{4}$. By applying the second mean-value theorem once more, we obtain

$$
I_{4}=\lambda\left\{[f(x+\delta)-f(x)] \int_{\delta}^{c} q_{0}(\lambda \eta) d \eta+[f(x+A)-f(x)] \int_{c}^{A} q_{0}(\lambda \eta) d \eta\right\},
$$

for some $\delta<c<A$. Thus, by taking the limit of (14) as $\lambda \rightarrow \infty$, we obtain by (13) that $I_{4} \rightarrow 0$ as $\lambda \rightarrow \infty$, and this completes the proof for case 1 .

In case $2, f \in L_{\text {loc }}^{1}(\mathcal{R})$ but is of bounded variation in a neighborhood of $\pm \infty$, and tends to zero as $|x| \rightarrow \infty$. Therefore, we write $I_{2}=I_{3}+I_{8}$, where $I_{3}$ is defined as before and

$$
I_{8}=\lambda \int_{\delta}^{\infty}[f(x+\eta)-f(x)] q_{0}(\lambda \eta) d \eta
$$

which we may write as

$$
I_{8}=\lim _{N \rightarrow \infty} I_{8}^{N}=\lim _{N \rightarrow \infty} \lambda \int_{\delta}^{N}[f(x+\eta)-f(x)] q_{0}(\lambda \eta) d \eta
$$

Showing that $I_{3}$ can be made arbitrarily small is the same as before. As for $I_{8}$, we have by the the second mean-value theorem once more that

$$
I_{8}^{N}=\lambda\left\{[f(x+\delta)-f(x)] \int_{\delta}^{B} q_{0}(\lambda \eta) d \eta+[f(x+N)-f(x)] \int_{B}^{N} q_{0}(\lambda \eta) d \eta\right\}
$$


for some $\delta<B<N$. Thus

$$
I_{8}^{N}=[f(x+\delta)-f(x)] \int_{\lambda \delta}^{\lambda B} q_{0}(y) d y+[f(x+N)-f(x)] \int_{\lambda B}^{\lambda N} q_{0}(y) d y .
$$

Taking the limits as $N \rightarrow \infty$ yields

$$
I_{8}=[f(x+\delta)-f(x)] \int_{\lambda \delta}^{\lambda B} q_{0}(y) d y-f(x) \int_{\lambda B}^{\infty} q_{0}(y) d y
$$

which, after taking the limits as $\lambda \rightarrow \infty$, yields that $I_{8} \rightarrow 0$, and this completes the proof of part (2).

To show that the continuity of a function at a point is not sufficient to ensure the pointwise convergence of its wavelet expansion, one needs only to observe that for Shannon's wavelet

$$
f_{m}(x)=f_{\lambda}(x)=\int_{-\infty}^{\infty} f(t) \frac{\sin \lambda \pi(x-t)}{\pi(x-t)} d t,
$$

which is Fourier's integral formula. It is well known that the mere continuity of a function at a point is not sufficient for the convergence of that integral; see [5].

If $\phi$ generates an orthogonal basis, then the condition $\phi_{k}^{*}(x)=O\left(k^{-\alpha}\right)$ is not needed since in this case $\phi_{k}(x)=\phi_{k}^{*}(x)=O\left(k^{-1}\right)$.

\section{REFERENCES}

[1] I. Daubechies, Ten Lectures on Wavelets, SIAM Publications, Soc. Indust. Appl. Math., Philadelphia (1992). MR 93e:42045

[2] S. Kelly, M. Kon, and L. Raphael, Pointwise convergence of wavelet expansions, Bull. Amer. Math. Soc., Vol. 30 (1994), pp. 87-94. MR 95a:42048

[3] S. Kelly, M. Kon, and L. Raphael, Local convergence for wavelet expansions, J. Funct. Anal., Vol. 126 (1994), pp. 102-138. MR 95k:42048

[4] Y. Meyer, Ondelettes, Herman, Paris (1990).

[5] E. Titchmarsh, Introduction to the Theory of Fourier Integrals, Oxford University Press, United Kingdom (1937).

[6] G. Walter, Pointwise convergence of wavelet expansions, J. Approx. Theory, Vol. 80 (1995), pp. 108-118. MR 96a:42056

[7] A. I. Zayed and G. Walter, Wavelets in closed forms, to appear.

Department of Mathematics, University of Central Florida, Orlando, Florida 32816

E-mail address: zayed@pegasus.cc.ucf.edu 